\title{
Sistema tutorial de aprendizaje y nivelación para aspirantes a PMP
}

\author{
VÍCTOR DANIEL GIL-VERA ${ }^{a}$
}

pp. 7-14
RESUMEN La certificación Project Management Professional (PMP) permite a una persona desempeñar su labor y ser reconocida como directora profesional de proyectos; sirve para demostrar a empleadores, clientes y profesionales en un área, que se tiene el conocimiento, la experiencia y las habilidades necesarias para dirigir proyectos y lograr resultados satisfactorios. Este artículo tiene como objetivo desarrollar un sistema tutorial de aprendizaje para dispositivos móviles que sirva como herramienta de preparación a todos los aspirantes a la certificación PMP otorgada por el Project Management Institute (PMI). Gracias a las tecnologías de la información y la comunicación (TIC), en la actualidad existen numerosas herramientas y estrategias de enseñanza que involucran el uso de recursos electrónicos y digitales (M-learning, B-learning, E-learning, etc.) para dinamizar y hacer más efectivos los procesos de enseñanza-aprendizaje. Con el desarrollo de este trabajo se concluye que los sistemas tutoriados (ST) facilitan el proceso de aprendizaje y permiten transformar el modelo educativo tradicional en un modelo versátil, flexible y didáctico.

PALABRAS CLAVE gestión de proyectos, M-learning, PMP, sistemas tutoriados de aprendizaje, TIC.

\section{HISTORIA DEL ARTÍCULO}

¿CÓMO CITAR?:

Gil-Vera, V. (2016). Sistema tutorial de aprendizaje y nivelación para aspirantes a PMP. Perspectiva Empresarial, 3(1), 7-14. http:// dx.doi.org/10.16967\%2Frpe.v3n1a6

RECIBIDO: 8 de octubre de 2015 APROBADO: 25 de enero de 2016

CORRESPONDENCIA:

Víctor Daniel Gil-Vera, Carrera 82 $\mathrm{N}^{\circ}$ 50-42 Calasanz - Medellín Antioquia - Colombia

a Magíster en Ingeniería de sistemas, docente investigador de la Fundación Universitaria Luis Amigó, Centro de Investigaciones, Provincia San José, Colombia. Correo electrónico: victor.gilve@amigo.edu.co 
¿CÓMO CITO EL ARTÍCULO? HOW TO CITE THIS PAPER?

CHICAGO:

Gil-Vera, Víctor Daniel. 2016. "Sistema tutorial de aprendizaje y nivelación para aspirantes a PMP". Perspectiva Empresarial 3(1): 7-14. http:// dx.doi.org/10.16967\%2Frpe. v3n1a6

MLA:

Gil-Vera, Víctor Daniel. "Sistema tutorial de aprendizaje y nivelación para aspirantes a PMP". Perspectiva Empresarial 3.1 (2016): 7-14. Digital. http://dx.doi. org/10.16967\%2Frpe.v3n1a6

\section{TUTORIAL LEARNING AND UPDATING SYSTEM PMP ASPIRANTS}

ABSTRACT The Project Management Professional (PMP) certification allows a person to carry out their work and be recognized as a professional project manager. This certificate; also helps to demonstrate knowledge, experience and skills to manage projects and achieve successful skills before employers, clients and professionals in a specific field. This paper aims to develop a tutorial learning system for mobile devices, to be used as a preparation tool for all candidates to the PMP certification with the Project Management Institute (PMI). Thanks to the information and Communications Technologies (ICTs), there are now a number of tools and teaching strategies related to the use of electronic and digital resources (M-learning B-learning, E-learning, etc.) to streamline and make teaching-learning processes more effective. This paper concludes that tutorial-based systems (ST) facilitate the learning process and can turn the traditional educational approach into a versatile, flexible and didactic model.

KEYWORDS project management, M-learning, PMP, tutored learning systems, ICT.

\section{SISTEMA TUTORADO DE APRENDIZADO E NIVELAÇÃO PARA ASPIRANTES À PMP}

RESUMO A certificação Project Management Professional (PMP) permite a uma pessoa desempenhar sua labor e ser reconhecida como diretora profissional de projetos; serve para mostrar aos empregadores, clientes e profissionais numa área, que ela tem o conhecimento, a experiência e as habilidades necessárias para dirigir projetos e conseguir resultados satisfatórios. Este artigo tem como objetivo desenvolver um sistema tutorial de aprendizado para dispositivos móveis que funcione como ferramenta de preparação para tudo aspirante à certificação PMP outorgada pelo Project Management Institute (PMI). Graças às tecnologias da informação e comunicação (TIC), na atualidade existem distintas ferramentas e estratégias de ensino que envolvem o uso dos recursos digitais (M-learning, B-learning, E-learning, etc.) para dinamizar e tornar mais eficientes os processos de ensino-aprendizado. Este trabalho conclui que os sistemas tutorados (ST) facilitam o processo de aprendizado e permitem transformar o modelo educativo tradicional num modelo versátil flexível e didático.

PALAVRAS CHAVE Gestão de projetos, M-learning, PMP, sistemas tutorados de aprendizado, TIC. 


\section{Introducción}

Los sistemas tutoriados (ST) son una rama de la inteligencia artificial aplicada (IA), cuyo principal objetivo es simular el proceso de enseñanza de un experto en un área específica de conocimiento, tanto en el dominio de un tema específico como en los aspectos relacionados con la pedagogía y la comunicación con el usuario. Los ST están diseñados para guiar el proceso de aprendizaje de un usuario en cualquier momento y lugar, sin la necesidad de interactuar directamente con un experto.

El sistema que se presenta en este trabajo fue diseñado para facilitar el proceso de enseñanza aprendizaje en los cinco grupos de procesos de la dirección de proyectos: iniciación, planificación, ejecución, seguimiento-control y cierre, y en las diez áreas de conocimiento: gestión de la integración, alcance, tiempo, costos, calidad, recursos humanos, comunicaciones, riesgos, adquisiciones e interesados. El sistema está conformado por cuatro grandes módulos de aprendizaje; en cado uno se presentan los fundamentos y nociones básicos, actividades interactivas, casos de aplicación reales y preguntas situacionales, de conocimiento, de interpretación, de fórmulas, de técnicas específicas y de conocimiento del PMPBOK, que sirven de preparación para realizar la prueba que otorga la certificación PMP.

Con el desarrollo de este trabajo se concluye que los ST permiten transformar el modelo educativo tradicional de enseñanza - aprendizaje, convirtiéndolo en un modelo versátil, flexible y didáctico tanto para el docente como para el estudiante, facilitando el proceso de aprendizaje en cualquier área de conocimiento.

\section{Objetivo}

Desarrollar un sistema tutorial ST de aprendizaje para dispositivos móviles que reúna las temáticas y conceptos teóricos fundamentales establecidos por el Project Management Institute (PMI), que sirva de herramienta para todos los aspirantes a la certificación PMP.

\section{Sistemas tutoriados inteligentes}

Los sistemas tutoriados (ST) fueron utilizados por primera vez en los años 1970, como una manera de proporcionar una mayor flexibilidad a la estrategia de aprendizaje y de lograr una mejor interacción con el usuario (Aguilar et al., 2011). Los ST han proporcionado un terreno fértil para investigaciones en inteligencia artificial (IA) a lo largo de los últimos veinticinco años (Corbett \& Koedinger, 1997). El objetivo principal de los ST es capturar el conocimiento de expertos para crear interacciones dinámicas con usuarios, permitiéndoles identificar las fortalezas y debilidades sin tener que interactuar directamente con un experto humano (Aguilar et al., 2011).

Los ST son sistemas de enseñanza basados en computadores que cuentan con modelos de instrucción de contenido que especifican qué y cómo enseñar (Murray, 2003). Estos, tienen la capacidad de hacer inferencias sobre el dominio de temáticas con el fin de adaptar dinámicamente su contenido, permitiendo a los estudiantes "aprender haciendo" en contextos realistas y significativos, y tener más control sobre su aprendizaje (Murray, 2003). La principal ventaja de los ST, si se comparan con los sistemas tutoriales tradicionales, radica en su flexibilidad, tanto en el enfoque como en la adaptación al estudiante. Los sistemas tutoriales tradicionales contienen una gran cantidad de reglas e información, lo que puede generar confusión (Aguilar et al., 2011).

El diseño de los ST se basa en el supuesto fundamental de que los estudiantes aprenden mejor en simulaciones de un contexto real en que puedan usar sus conocimientos es decir, aprenden haciendo, cometiendo errores y construyendo conocimiento (Ferreira \& Atkinson, 2009). En resumen, los ST tienen el potencial de introducir avances en el ámbito del aprendizaje y la educación (Ramesh \& Rao, 2012) y permiten que el aprendizaje pueda realizarse en cualquier momento y lugar (Kalhoro, 2010).

\section{Aplicaciones M-learning para la formación de PMP: trabajos relacionados}

En esta sección se presenta el método de búsqueda utilizado para identificar trabajos relacionados con aplicaciones móviles para la formación y capacitación de Profesionales en Dirección de Proyectos (PMP). Se realizó una búsqueda detallada de revisiones de literatura, artículos, resúmenes de conferencias y publicaciones en bases de datos especializadas.

Adicionalmente, se revisaron artículos publicados en las siguientes revistas científicas: Journal 
of Computer Assisted Learning (JCAL), Computers and Education, Journal of the Learning Sciences, International Journal of Mobile Learning and Organisation, International Journal of ComputerSupported Collaborative Learning e International Journal of Learning Technology.

El rango de fechas va desde el año 2010 hasta el 2015. En total, se inspeccionaron 54 publicaciones de conferencias de M-learning y 10 artículos de revistas especializadas. Las palabras de búsqueda utilizadas fueron: Intelligent Tutoring Systems, Project Management Professional, PMI, M-learning applications. En total se estudiaron 64 publicaciones. Las ecuaciones de búsqueda utilizadas fueron:

1. (TITLE-ABS-KEY (M-learning) OR (mobile learning) AND (C(Projects Management professional) OR (PMI) ) OR ( ( (Management) OR ( (Certification) OR (applications) ) ) ) >2009

2. (TITLE-ABS-KEY (M-learning) AND (mobile learning) AND (C(Projects Management professional) OR (PMI) ) AND ( ( (Management) AND ( (Certification) AND (applications) ) ) ) $>2009$

3. (TITLE-ABS-KEY (M-learning) AND (mobile learning) AND (C(Projects Management professional) OR (PMI) ) OR ( ( (Management) AND ( (Certification) OR (applications) ) ) ) $>2009$

4. (TITLE-ABS-KEY (M-learning) AND (mobile learning) AND (C(Projects Management professional) OR (PMI) ) AND ( ( (Management) AND ( (Certification) OR (applications) ) ) ) $>2009$

5. (TITLE-ABS-KEY (M-learning) OR (mobile learning) OR ( ((Projects Management professional) OR (PMI) ) OR ( ( (Management) OR ( (Certification) AND (applications) ) ) ) >2009

De los resultados de la búsqueda se encontró que la mayoría de las aplicaciones desarrolladas para dispositivos móviles que sirven de ayuda para la preparación de la prueba que otorga la certificación PMP, únicamente presentan teoría relacionada con los grupos de procesos y áreas de conocimiento establecidos en la guía PMBOK, no cuentan con recursos ni actividades interactivas que permitan al usuario identificar sus fortalezas y debilidades, y únicamente funcionan para dispositivos con sistema operativo Android.

\section{Metodología}

La metodología empleada para la construcción de "Cyber-PMP" fue la revisión sistemática de literatura (RSL), proceso sistemático, auditable y organizado que busca responder a una o más preguntas de investigación, tomando como base publicaciones previas (Kitchenham, 2004). Una RSL es un medio para identificar, evaluar e interpretar toda la investigación pertinente, disponible y relacionada con una pregunta de investigación, área temática o fenómeno de interés. Los estudios individuales que sirven de base para la realización de una RSL se denominan estudios primarios; la RSL por sí sola se considera como un estudio secundario (Staples \& Niazi, 2007). Según Kitchenham (2004), los pasos que se deben seguir para la realización de una RSL son los siguientes:

- Identificación de investigación: el objetivo de una RSL es encontrar la mayor cantidad posible de estudios primarios relacionados con una pregunta de investigación, tomando como base criterios y parámetros de búsqueda antes de hacer la selección final. La definición de estos criterios es la principal diferencia que la distingue de la revisión tradicional de literatura.

- Selección de estudios: una vez obtenidos los estudios primarios potencialmente relevantes, se debe evaluar su calidad real para filtrar los estudios que realmente puedan contribuir a responder la(s) pregunta(s) de investigación.

- Extracción de datos: el objetivo de esta etapa es diseñar formularios de extracción de datos para registrar con precisión la información que los investigadores obtienen de los estudios primarios. Los formularios de extracción de datos deben ser definidos al momento de determinar el protocolo de estudio.

- Síntesis: consiste en recopilar y resumir los resultados de los estudios primarios seleccionados. La síntesis debe ser descriptiva (no cuantitativa). Sin embargo, a veces es posible complementar una síntesis descriptiva con un resumen cuantitativo.

La pregunta de investigación considerada en este trabajo fue:

P1. ¿Cuáles son las principales temáticas, principios y metodologías que deben conocer y dominar 
los aspirantes a la certificación Profesional en Dirección de Proyectos del PMI (PMP)?

Las palabras de búsqueda utilizadas fueron: Intelligent Tutoring Systems, Project Management Professional, PMI, M-learning applications, PMP. Adicionalmente, se realizaron búsquedas con estas palabras mezcladas entre sí. La ecuación de búsqueda utilizada fue:

(1) TITLE-ABS-KEY (ITS, Project Management Professional, PMI, M-learning applications PMP) AND PUBYEAR > 2009 AND (LIMIT-TO (SUB)-AREA, "BUSI") OR LIMIT-TO ( SUBJ-AREA, "ECON")

El rango de fechas de publicación consideradas en la revisión del estado del arte va desde el año 2010 hasta el año 2015. En total se recopilaron 22 publicaciones entre artículos, artículos de conferencias, resúmenes, capítulos de libros y artículos en desarrollo. En la tabla 1 se presenta el número de publicaciones por tipo recopiladas en la revisión del estado del arte.

TABLA 1. Número de publicaciones por tipo

\begin{tabular}{lc}
\hline TIPO DE PUBLICACIÓN & CANTIDAD \\
\hline Artículos & 13 \\
\hline Artículos de conferencias & 5 \\
\hline Capítulos de libros & 1 \\
\hline Libros & 1 \\
\hline Resúmenes & 2 \\
\hline Total & $\mathbf{2 2}$ \\
\hline
\end{tabular}

Fuente: elaboración propia.

Adicionalmente, se realizó una búsqueda de literatura gris, se recopilaron 17 manuales y 12 cartillas electrónicas sobre gestión y administración de proyectos, publicados por el Project Management Institute (PMI), por la Escuela de Dirección y Administración de Proyectos (EDAP) y por la Escuela de Ingeniería de Antioquía (EIA), los cuales fueron utilizados para la construcción de la base de conocimientos (BC) del sistema tutoriado desarrollado.

"Cyber-PMP" se desarrolló con el software XOT, que proporciona un conjunto completo de herramientas de código abierto para desarrolladores y creadores de materiales didácticos interactivos (Xerte, 2015). Está orientado a desarrolladores de contenido interactivo e-learning y permite crear contenidos sofisticados con algunas secuencias de comandos (Xerte, 2015).

\section{Caso de aplicación}

Debido a la gran cantidad de temáticas que deben conocer y dominar los aspirantes a la certificación PMP, se consideró oportuno desarrollar un sistema tutoriado (ST) que permitiera reforzar y afianzar los conocimientos básicos establecidos en las guías del PMI. Dado que las temáticas en las que más se presentan dificultades al momento de realizar la prueba están relacionadas con preguntas situacionales que involucran la toma de decisiones, el sistema presenta casos reales que le dan la oportunidad al usuario de demostrar su experiencia, habilidades y dominio de las temáticas fundamentales de dirección de proyectos. "Cyber-PMP" permite al usuario adquirir los conocimientos básicos que componen un curso de preparación para la certificación PMP, independientemente del área y del nivel profesional del usuario.

\section{Principales elementos de "Cyber-PMP"}

Base de conocimientos (BC): reúne toda la información sobre los conceptos básicos de gestión de proyectos, grupos de procesos y áreas de conocimiento (Entradas, Herramientas y Técnicas, Salidas). La Tabla 2, presenta el contenido de la base de conocimientos (BC) de "Cyber-PMP":

\section{Funcionamiento de "Cyber-PMP”.}

"Cyber-PMP" funciona para dispositivos móviles con sistemas operativos Android y iOS con acceso a internet. Además, puede ser utilizado en ordenadores personales y portátiles. Se puede acceder desde cualquier navegador (Google Chrome, Mozilla Firefox, Opera, Safari, Internet Explorer). El dispositivo móvil u ordenador debe tener instalada la versión 11.1 de Adobe Flash Player. Para utilizarlo, se debe acceder al siguiente enlace: https://www.dropbox.com/sh/gd4nfinco9xusph /AAB8PJIewtVhc6VKdWGKlpIqa?dl=0

El usuario debe extraer el contenido del enlace anterior y ejecutar el archivo denominado "Index. htm". Una vez el sistema empieza a funcionar, el usuario debe seleccionar el área de procesos que desea conocer: iniciación, planificación, ejecución, monitoreo y control, o cierre. Posteriormente, dependiendo del área seleccionada, "Cyber-PMP" presenta cada una de las diez áreas de conocimiento, de las cuales el usuario debe seleccionar una. En cada una de ellas se encuentran los conceptos 
TABLA 2. Contenidos Base de conocimientos (BC)

\begin{tabular}{lll}
\hline TIPO DE PUBLICACIÓN & ÁREAS DE CONOCIMIENTO & HERRAMIENTAS \\
\hline Fundamentos & Integración & Recopilar información \\
\hline Iniciación & Alcance & Controlar y verificar el desempeño \\
\hline Planificación & Tiempo & Desarrollar el cronograma \\
\hline Ejecución & Costos & Estimar tiempo y costo \\
\hline Seguimiento y control & Calidad & Priorización \\
\hline & Recursos Humanos & \\
\cline { 2 - 2 } Cierre & Comunicación & \\
\cline { 2 - 3 } & Riesgos & Interesados \\
\cline { 2 - 3 } & Adquisiciones & \\
\hline
\end{tabular}

Fuente: elaboración propia.

y nociones fundamentales (entradas, herramientas y técnicas, y salidas), una serie de actividades que tienen como finalidad evaluar el nivel de dominio de las temáticas propuestas por el sistema, y un test que reúne diferentes tipos de preguntas permitiéndole conocer los aspectos que debe reforzar para tener un buen desempeño en la presentación de la prueba que otorga la certificación de Profesional en Dirección de Proyectos del PMI (PMP). La figura 1, presenta la pantalla inicial del sistema.

FIGURA 1. Interfaz inicial “Cyber-PMP”
La figura 2 muestra la segunda interfaz del sistema, donde se especifican los objetivos, la audiencia a la que va dirigida y cómo utilizarlo.

En la figura 3 se presenta la primera actividad propuesta por el sistema perteneciente al primer grupo de conocimientos (procesos de iniciación) y a la primera área de conocimiento (gestión de la integración del proyecto).

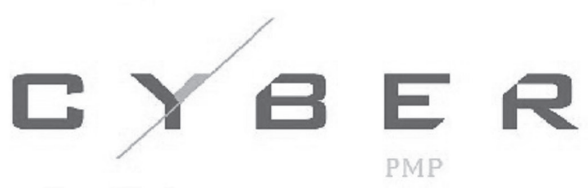


FIGURA 2. Segunda interfaz “Cyber-PMP”

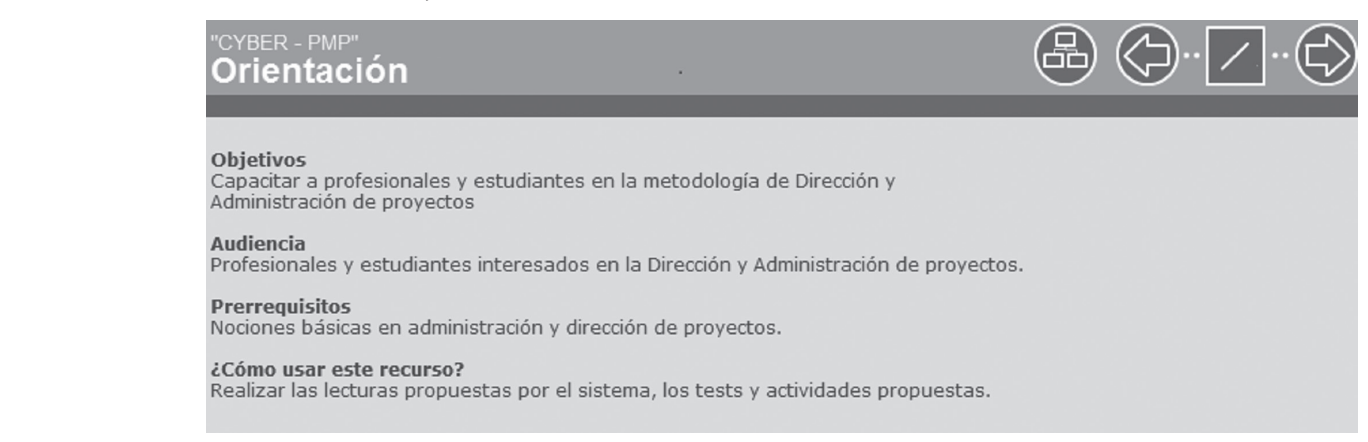

Fuente: elaboración propia.

FIGURA 3. Actividad 1 "Cyber-PMP”

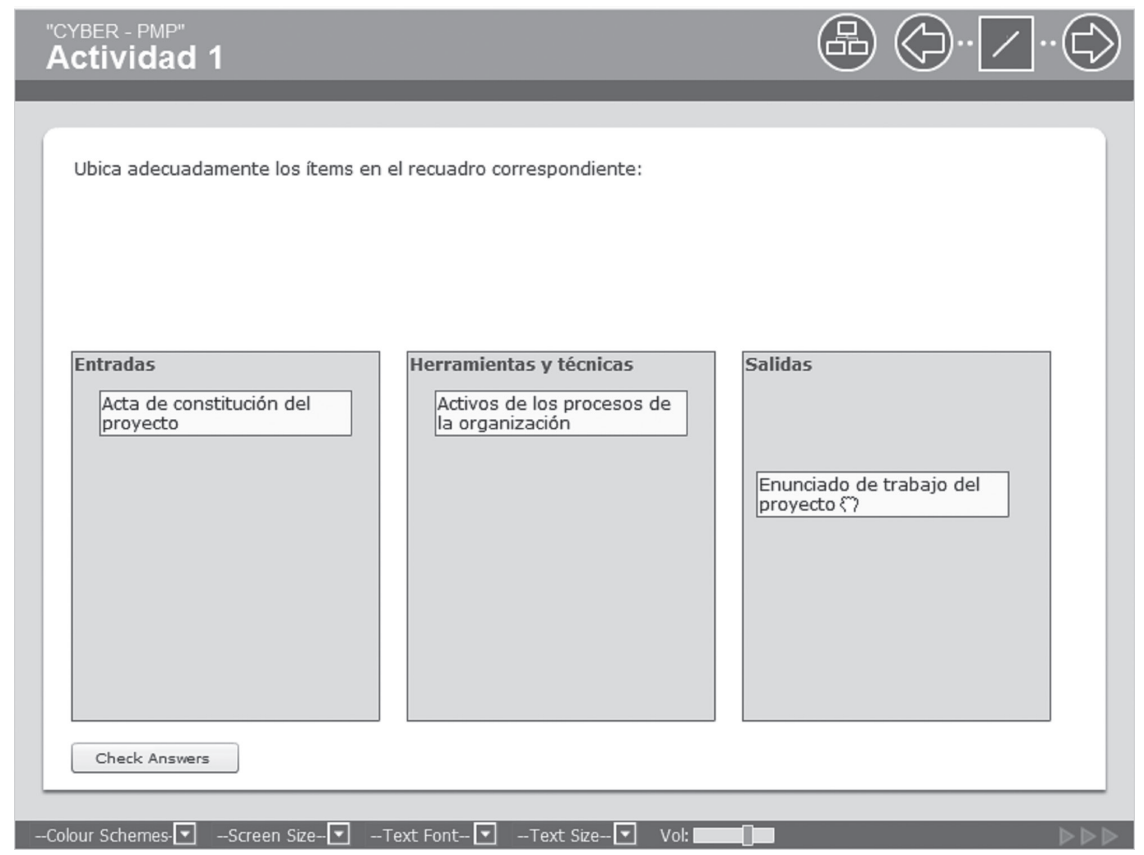

Fuente: elaboración propia.

\section{Conclusiones}

Los sistemas tutoriados (ST) facilitan el proceso de aprendizaje en cualquier área de conocimiento, ya que tienen la capacidad de retroalimentar y suministrar instrucciones individuales a los usuarios en tiempo real, sin requerir la intervención de un experto humano; así permiten transformar el modelo educativo tradicional de enseñanza - aprendizaje en un modelo versátil, flexible y didáctico tanto para el docente como para el estudiante.
En la actualidad, debido a la alta demanda de directores de proyectos especializados, la certificación PMP puede convertirse en un factor diferencial al momento de aspirar a empleos relacionados con gestión de proyectos, por lo que el uso de herramientas de aprendizaje para dispositivos móviles asociadas con el tema se constituye en una ventaja competitiva para todos los aspirantes a dicha certificación, ya que las pueden utilizar en cualquier momento y lugar, lo cual les permite optimizar tiempo y recursos. 


\section{REFERENCIAS}

Aguilar, R., Muñoz, V., González, E. J., Noda, M., Bruno, a., \& Moreno, L. (2011). Fuzzy and MultiAgent Instructional Planner for an Intelligent Tutorial System. Applied Soft Computing, 11(2), 2142-2150. doi:10.1016/j.asoc.2010.07.013

Corbett, A., \& Koedinger, K. (1997). Intelligent Tutoring Systems. In Handbook of Human-Computer Interaction (2nd ed., pp. 2-5). Pittsburgh: Elsevier Science B. V.

Ferreira, A., \& Atkinson, J. (2009). Designing a feedback component of an intelligent tutoring system for foreign language. Knowledge-Based Systems, 22(7), 496-501. doi:10.1016/j.knosys.2008.10.012

Kalhoro, Q. (2010). M - Learning an innovative advancement of ICT in education. 4th International Conference on Distance Learning and Education (ICDLE), pp 148-151.San Juan, PR.: IEEE. doi: 10.1109/ICDLE.2010.5606017

Kitchenham, B. (2004). Procedures for Performing Systematic Reviews. Keele: Keele University Technical Report TR/SE-0401.
Murray, T. (2003). An overview of intelligent tutoring system authoring tools : Updated Analysis of the State of the Art. In Authoring Tools for Advanced Technology Learning Environments (pp. 493-546). Springer Netherlands. doi 10.1007/978-94-0170819-7_17

PMI. (2012). Manual del PMP. Pennsylvania: Project Management Institute. Retrieved from http:// www.pmi.org/ /media/PDF/Certifications/ManualPMP_201204.ashx

Ramesh, V., \& Rao, N. (2012). Tutoring and Expert Modules of Intelligent Tutoring Systems. In IEEE Fourth International Conference on Technology for Education (pp. 251-252), Hyderabad: IEEE. doi 10.1109/T4E.2012.52

Staples, M., \& Niazi, M. (2007). Experiences using systematic review guidelines. Journal of Systems and Software, 80(9), 1425-1437.

Xerte. (2015). ¿Qué es xerte?. Retrieved August 31, 2015, from http://xerte.org.uk/index.php?lan$\mathrm{g}=\mathrm{es} \&$ Itemid $=642$ 